\title{
Dynamic graph cut based segmentation of mammogram
}

\author{
S. Pitchumani Angayarkanni ${ }^{1 *}$, Nadira Banu Kamal ${ }^{2}$ and Ranjit Jeba Thangaiya ${ }^{3}$
}

\author{
${ }^{*}$ Correspondence: \\ pitchu_mca@yahoo.com \\ ${ }^{1}$ Department of Computer \\ Science, Lady Doak College, \\ Madurai, Tamil Nadu, India \\ Full list of author information \\ is available at the end of the \\ article
}

\begin{abstract}
This work presents the dynamic graph cut based Otsu's method to segment the masses in mammogram images. Major concern that threatens human life is cancer. Breast cancer is the most common type of disease among women in India and abroad. Breast cancer increases the mortality rate in India especially in women since it is considered to be the second largest form of disease which leads to death. Mammography is the best method for diagnosing early stage of cancer. The computer aided diagnosis lacks accuracy and it is time consuming. The main approach which makes the detection of cancerous masses accurate is segmentation process. This paper is a presentation of the dynamic graph cut based approach for effective segmentation of region of interest (ROI). The sensitivity, the specificity, the positive prediction value and the negative prediction value of the proposed algorithm are determined and compared with the existing algorithms. Both qualitative and quantitative methods are used to detect the accuracy of the proposed system. The sensitivity, the specificity, the positive prediction value and the negative prediction value of the proposed algorithm accounts to 98.88 , 98.89, 93 and $97.5 \%$ which rates very high when compared to the existing algorithms.
\end{abstract}

Keywords: Fuzzification, Graph cut, Otsu's method and ROC

\section{Introduction}

The population based cancer registry evidently shows from the various statistics, that the incidence of breast cancer is rapidly rising, amounting to a significant percentage of all cancers in women. Breast cancer is the commonest cancer in urban areas in India and accounts for about $25-33 \%$ of all cancers in women. Over $50 \%$ of the breast cancer patients in India, being in stages 3 and 4 will definitely face the survival problem (Hassanien and Ali 2011). The survival rate can be increased only through early diagnosis. Image processing technique together with data mining is used for extraction and analysis of the ROI. Tumor can be classified into three categories normal, benign and malignant. A normal tumor is a mass of tissue which exists at the expense of healthy tissue. Malignant tumor has no distinct border. They tend to grow rapidly, increasing the pressure within the breast cells and can spread beyond the point from which they originate. Thus they grow faster than benign tumors and cause serious health problems if, left unnoticed. Benign tumors are composed of harmless cells and they have clearly defined borders. They can be completely removed and are unlikely to recur. MRI mammogram images taken after the appropriate segmentation of the tumor make classification of tumor into

(c) 2015 Angayarkanni et al. This article is distributed under the terms of the Creative Commons Attribution 4.0 International License (http://creativecommons.org/licenses/by/4.0/), which permits unrestricted use, distribution, and reproduction in any medium, provided you give appropriate credit to the original author(s) and the source, provide a link to the Creative Commons license, and indicate if changes were made. 
malignant, benign and normal a difficult task, due to complexity and variation in tumor tissue characteristics like its shape, size, grey level intensities and location. Effective segmentation techniques results in accurate classification of such cancerous masses.

\section{Data acquisition}

A database of 1,528 mammograms, originating from the mammography image analysis society (MIAS), digital database for screening mammography, University of South Florida DDSM Resource, LLNL/UCSF database (Lawrence Livermore National Laboratories (LLNL), University of California at San Francisco) and Nijmegen digital mammogram database were used for the study.

\section{Methodology}

\section{Image preprocessing and enhancement}

The main objective behind the preprocessing step is to enlarge the intensity difference between objects and background. Preprocessing technique increases the optical inspection of an image. The proposed approach improves the image data by suppressing unwanted distortions and enhance the important image features. This will produce reliable representations of breast tissue structures. The fuzzy transformation function for computing the fuzzy plane value $P$ is defined as follows:

- $\alpha=\min$

- $\beta 1=(\alpha+\gamma) / 2$

- $\beta 2=(\max +\gamma) / 2$

- $\gamma=\max / 2$

The histogram equalization of the gray levels in the original image can be characterized using five parameters: $(\alpha, \beta 1, \gamma, \beta 2$, max). The aim is to decrease the gray levels below $\beta 1$, and above $\beta 2$. Intensity levels between $\beta 1$ and $\gamma$, and $\beta 2$ and $\gamma$ are stretched in opposite directions towards the mean $\gamma$ (Fig. 1).

\section{Procedure:}

Step 1: Fuzzification:

The following fuzzy rules are used for contrast enhancement:

Rule-1:

If $\alpha \leq u_{i}<\beta 1$ then $P=2\left(\left(u_{i}-\alpha\right) /(\gamma-\alpha)\right)^{2}$

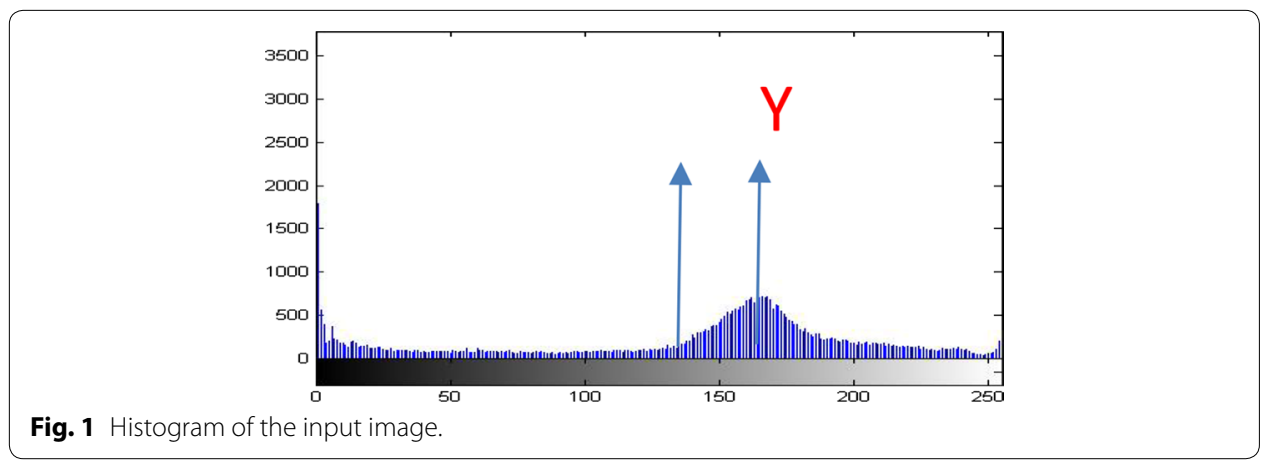


Rule-2:

If $\beta 1 \leq \mathrm{u}_{\mathrm{i}}<\gamma$ then $\mathrm{P}=1-2\left(\left(\mathrm{u}_{\mathrm{i}}-\gamma\right) /(\gamma-\alpha)\right)^{2}$

Rule-3:

If $\gamma \leq u_{i}<\beta 2$ then $P=1-2\left(\left(u_{i}-\gamma\right) /(\max -\gamma)\right)^{2}$

Rule-4:

If $\beta 2 \leq \mathrm{u}_{\mathrm{i}}<\max$ then $\mathrm{P}=2\left(\left(\mathrm{u}_{\mathrm{i}}-\gamma\right) /(\max -\gamma)\right)^{2}$

where $u_{i}=f(x, y)$ is the ith pixel intensity

Step 2: Fuzzy Modification

Step 3: Defuzzification

The quality of the preprocessed image is to be checked with the following parameters like peak signal to noise ratio (PSNR), noise standard deviation (NSD), mean square error (MSE), equivalent number of looks (ENL).

\section{Image segmentation and ROI extraction}

The region of interest i.e. the tumor region is segmented using the Graph cut method. The main purpose of using this method for segmentation is that it segments the mammogram into different mammographic densities. It is useful for risk assessment and quantitative evaluation of density changes. Apart from the above advantage it produces the contour (closed region) or a convex hull which is used for analyzing the morphological and novel features of the segmented region. The above technique results in efficient formulation of attributes which helps in classification of the ROI into benign, malignant or normal. Graph cuts have been used in recent years for interactive image segmentation (Hassanien and Badr 2003). The core ideology of graph cuts is to map an image onto a network graph, and construct an energy function on the labeling, and then do energy minimization with dynamic optimization techniques. This study proposes a new segmentation method using iterated graph cuts based on multi-scale smoothing. The multi-scale method can segment mammographic images with a stepwise process from global to local segmentation by iterating graph cuts. The modified graph cut approach used by K. Santle Camilus (Hassanien and Badr 2003) is implemented in this project.

Steps involved in graph cut segmentation are:

1. Form a graph

2. Sort the graph edges

3. Merging regions based on threshold

From the mammogram image a graph $G=(V, E)$ is constructed such that $V$ represents the pixel values of the $3 \times 3$ image and $E$ represents the edges defined between the neighboring pixels. The weight of any edge $\mathrm{W}(\mathrm{Vi}, \mathrm{Vj})$ is a measure of dissimilarity between the pixels $\mathrm{Vi}$ and $\mathrm{Vj}$. The weight for an edge is measured by means of considering the Euclidian distance between the two pixels Vi and Vj (Ertas et al. 2001; Shah et al. 2011; Masek et al. 2001; Thamaraichelvi and Yamuna 2013; Jayadevappa et al. 2009; Benfield et al. 2007; Elnakib et al. 2011). It is represented by the equation 


$$
\begin{aligned}
& \mathrm{W}(\mathrm{Vi}, \mathrm{Vj})=\sqrt{(\boldsymbol{x} \boldsymbol{i}-\boldsymbol{x} \mathbf{j})^{2}+(\boldsymbol{y} \boldsymbol{i}-\boldsymbol{y} \boldsymbol{j})^{2}} \\
& \mathrm{Vi}=(\mathrm{xi}, \mathrm{yi}) \quad \mathrm{Vj}=(\mathrm{xj}, \mathrm{yj})
\end{aligned}
$$

\section{Procedure:}

1. Sort the edges in ascending order of their weights such that $W\left(e_{1}\right) \leq W\left(e_{2}\right)$.

2. Pick one edge $e_{i}$ in the sorted order from $e_{i}$ to $e_{n}$ where $e_{i}$ is between two groups of pixels which determines whether to merge the two groups of pixel to form a single group or not. Each vertex is considered as a group. The two groups which satisfies the merge criteria are merged together. The different groups of pixels representing different regions or objects are obtained.

3. Determining the merge criteria: When the pixels of a group have intensity values similar to the pixels of the other group, then intuitively the calculated IRM between these groups should be small. The expected smaller value of the IRM to merge these two regions is tested by comparing it with the dynamic threshold. Hence, the merge criterion, to merge the two regions, $R_{1}$ and $R_{2}$, is defined as:

$\operatorname{Merge}\left(\mathrm{R}_{1}, \mathrm{R}_{2}\right), \quad$ if $\operatorname{IRM}\left(\mathrm{R}_{1}, \mathrm{R}_{2}\right) \leq D T(\mathrm{R} 1, R 2)$

Figure 2 specifies the weighted calculation applied to the input image. Figure 3 shows how graph cut method is applied on a $3 \times 3$ image. Figure 4 shows the stage by stage output of the proposed method and the segmented region is shown in Fig. 5.

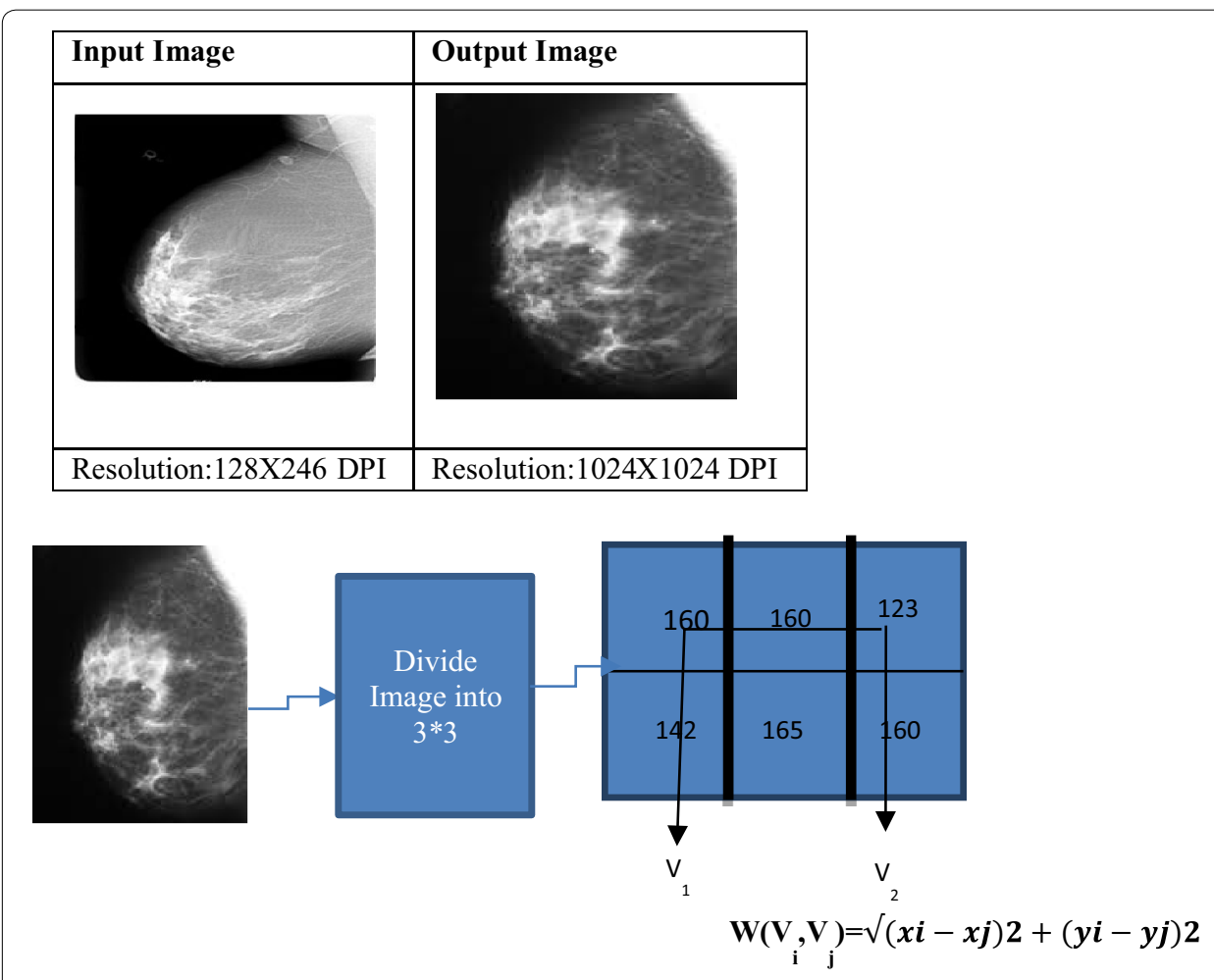

Fig. 2 Weight calculation for the $3 \times 3$ matrix. 

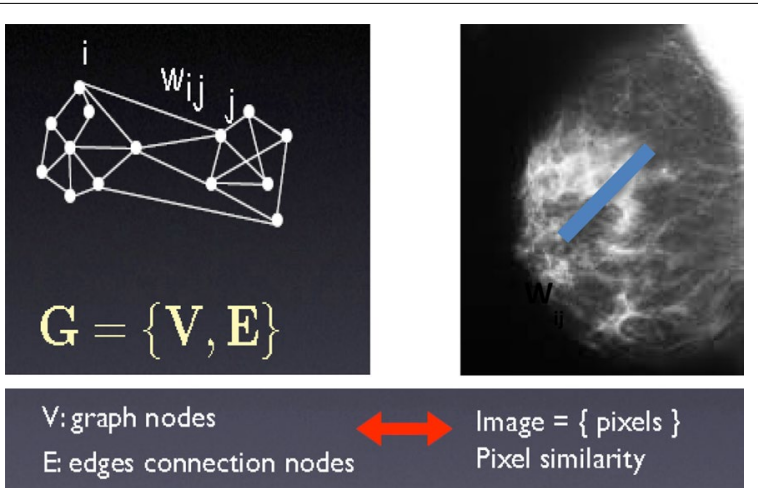

Fig. 3 Graph cut approach.

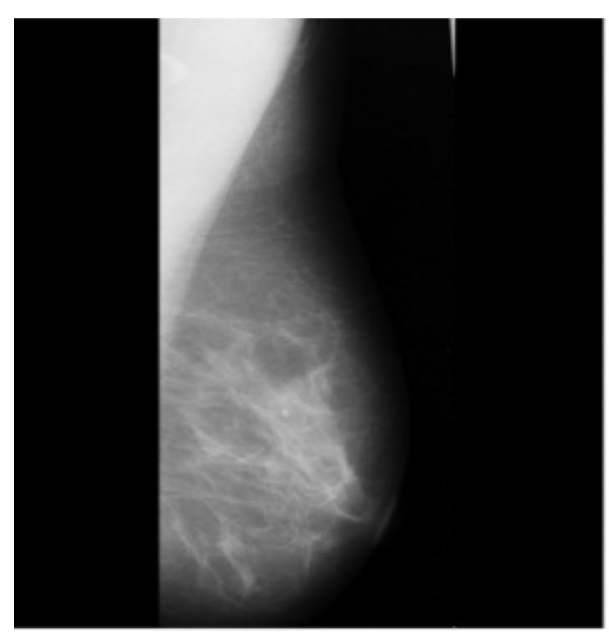

a

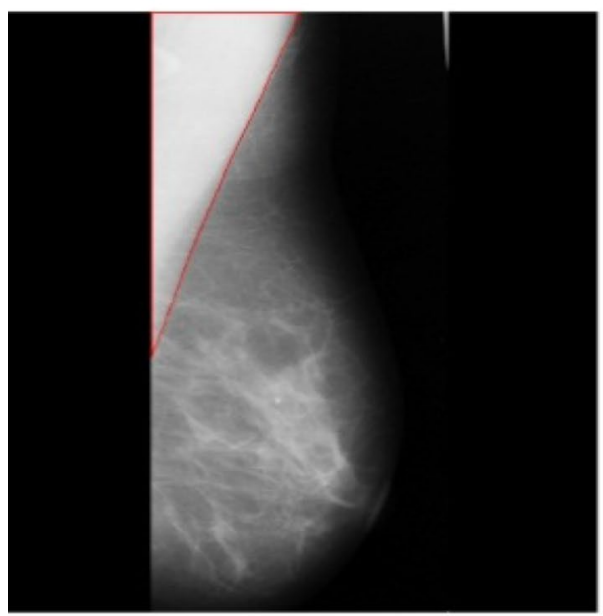

e

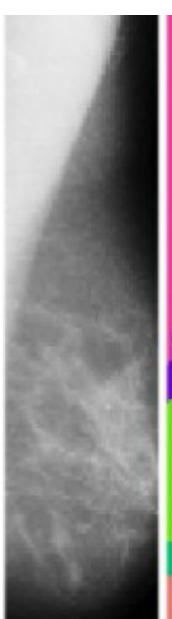

b

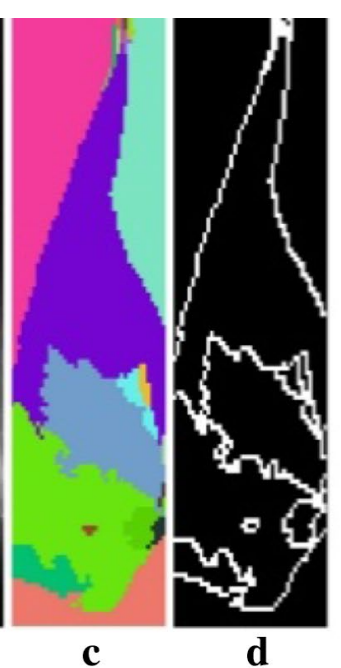

c

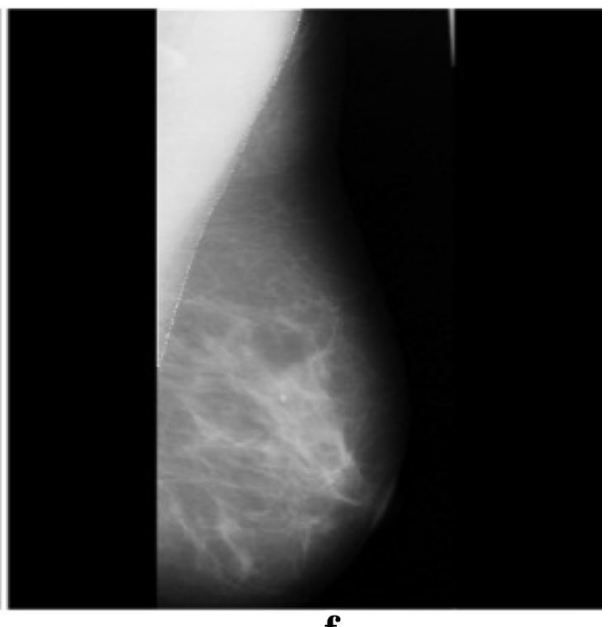

f

Fig. 4 a Input image, $\mathbf{b} \mathrm{ROI}$, c segmented boundaries, $\mathbf{d}$ edge, e pectoral muscle identification indicated by red color, $\mathbf{f}$ ground truth value represented by white. 


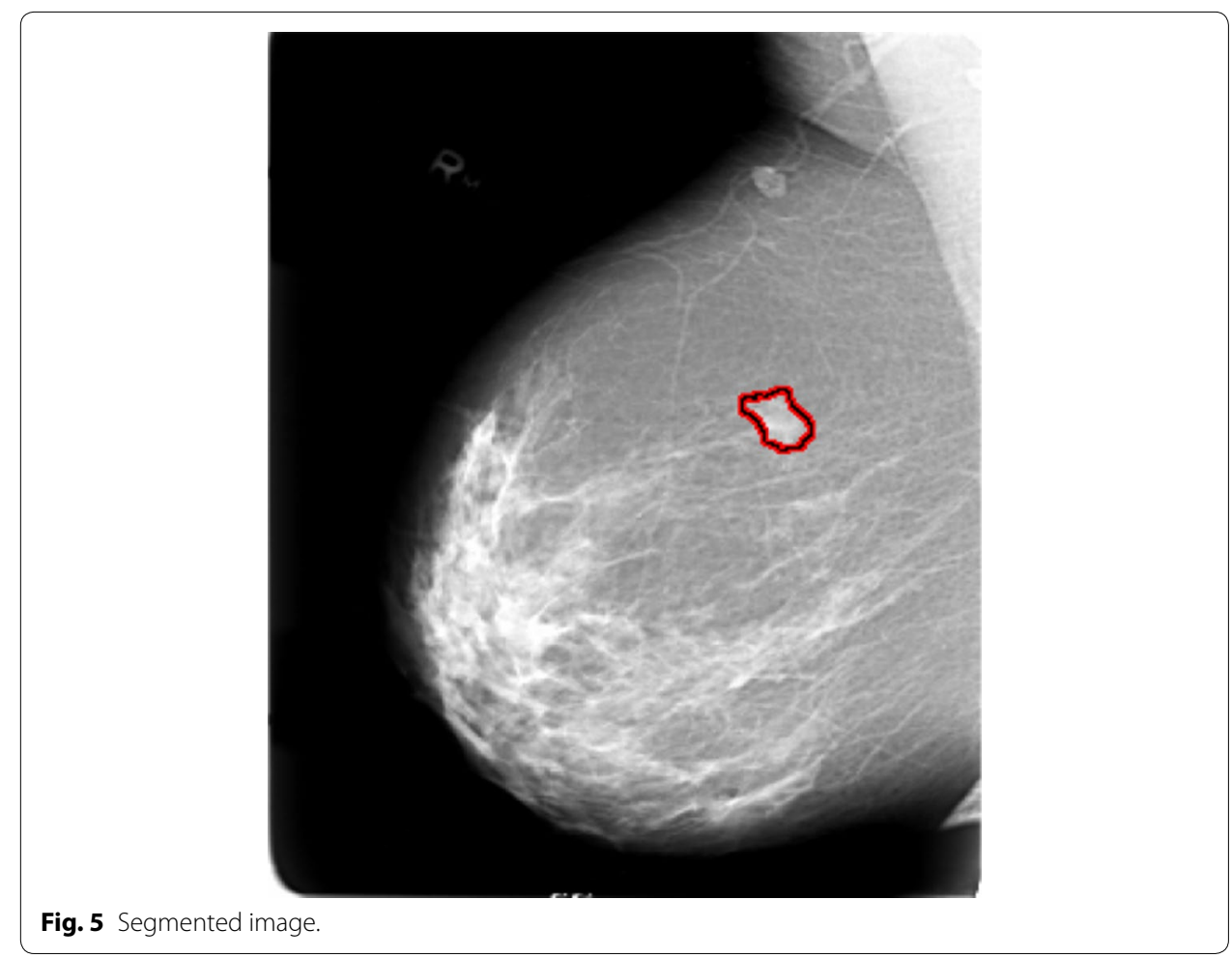

\section{Performance analysis}

Performance measure of the proposed mathematical approach at each stage was estimated.

\section{Preprocessing}

Tabulation in Table 1 clearly shows a high PSNR value which shows that the image is highly enhanced (Camilus et al. 2010).

\section{Segmentation}

The Table 2 below depicts the interpretation between the two approaches using the quantitative measures to determine the overall classification accuracy (Zhang et al. 2012; Annamalai et al. 2009; Ramaswamy and Rose 2009; Peng et al. 2010; Artan et al. 2012).

\section{Table 1 PSNR tabulation}

\begin{tabular}{llllll}
\hline PSNR & RMS & H & $\boldsymbol{\gamma}$ & MSE & Nature of filter \\
\hline 87.65 & 2.97 & 0.2111 & 0.0086 & 8.83 & FHQ \\
\hline
\end{tabular}

Table 2 Segmentation technique comparision

\begin{tabular}{lcc}
\hline Parameters & Hassanien method & Proposed method \\
\hline Target to background contrast measure based on standard deviation & 0.71 & 0.83 \\
Target to background contrast measure based on entropy & 0.76 & 0.90 \\
Index of fuzziness & 0.2892 & 0.010 \\
Fuzzy entropy & 0.1056 & -0.001 \\
PSNR & 86.75 & 90.88
\end{tabular}




\section{Segmentation accuracy}

Segmentation accuracy is depicted in Table 3.

\section{Computational efficiency}

Table 4 clearly depicts the computational efficiency of the proposed method is efficient compared to the other existing technique.

\section{Metrics for evaluating the segmentation technique includes}

The region-based criteria mutually compare the machine segmented regions with the correct ground truth regions.

Let $\mathrm{A}(\mathrm{I}, \mathrm{J})$ denote the machine segmented region and $\mathrm{B}(\mathrm{I}, \mathrm{J})$ denotes the ground truth region then the region overlap acceptance is controlled by the threshold $\mathrm{k}=0.75$ then

Region overlap Local refinement error

$$
\mathrm{E}(\mathrm{A}, \mathrm{B}, \mathrm{k})=|\mathrm{R}(\mathrm{A}, \mathrm{k}) / \mathrm{R}(\mathrm{B}, \mathrm{k})| / \mathrm{R}(\mathrm{A}, \mathrm{k})
$$

Edgel matching Overlay the original with segmented image and compute correspondence via min-cost assignment on bipartite graph.

The F-measure value is shown in Fig. 6.

\section{Conclusions}

The proposed mathematical approach yields a high level of accuracy within a minimum period of time that confirms the efficiency of the algorithm. The GUI based CAD system was developed using Scilab and R2. The segmentation speed accounts to 6 ms using graph cut based Otsu's thresholding. The main goal of classifying the tumors into benign, malignant and normal is achieved with a great accuracy compared to other techniques

Table 3 Segmentation accuracy metrics

\begin{tabular}{ll}
\hline Specificity & $95.5 \%$ \\
Sensitivity & $97.3 \%$ \\
Positive prediction value & $89 \%$ \\
Accuracy & $98.9 \%$ \\
Area under curve & 0.98 \\
Negative prediction value & $98 \%$
\end{tabular}

Table 4 Computational efficiency of the proposed method

\begin{tabular}{|c|c|c|c|}
\hline Methods & References & System specification & $\begin{array}{l}\text { Computational } \\
\text { time based on } \\
\text { implementation }\end{array}$ \\
\hline Rough set approach & Hassanien and Ali (2011) & \multirow{5}{*}{$\begin{array}{l}\text { Intel Pentium }{ }^{\circledR} \\
\text { CPU B950 Processor } \\
2 \text { GB RAM } \\
\text { 32-bit OS } \\
\text { Windows } 7\end{array}$} & $2^{\prime} 19^{\prime \prime}$ \\
\hline $\begin{array}{l}\text { Mathematical } \\
\text { Morphological }\end{array}$ & Bojar and Nieniewski (2008) & & $2^{\prime} 50^{\prime \prime}$ \\
\hline Shape and texture feature & Zakeri et al. (2012) & & $8^{\prime} 21^{\prime \prime}$ \\
\hline $\begin{array}{l}\text { Shape, edge-sharpness, } \\
\text { and texture features }\end{array}$ & Mu et al. (2008) & & $0^{\prime} 45^{\prime \prime}$ \\
\hline Proposed method & Angayarkanni et al. (2002) & & $0^{\prime} 03^{\prime \prime}$ \\
\hline
\end{tabular}




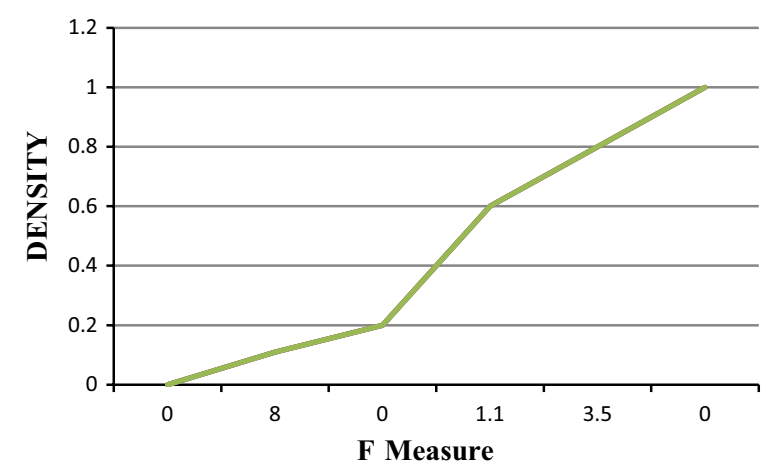

Fig. 6 F-measure.

because of the implementation of the accurate segmentation technique employed. The proposed technique is computationally efficient as specified in the tabulation above. Further the complexity of the algorithm in asymptotic sense is equivalent to o $(\log n)$.

\section{Authors' contributions}

A mathematical model for effective detection and segmentation of cancerous masses has been proposed. All authors read and approved the final manuscript.

\section{Author details}

1 Department of Computer Science, Lady Doak College, Madurai, Tamil Nadu, India. ${ }^{2}$ Department of M.C.A., TBAK College, Kilakarai, Ramnad, Tamil Nadu, India. ${ }^{3}$ Department of M.C.A., Karunya University, Coimbatore, Tamil Nadu, India.

\section{Acknowledgements}

Nil.

\section{Compliance with ethical guidelines}

\section{Competing interests}

The authors declare that they have no competing interests.

Received: 22 July 2015 Accepted: 23 July 2015

Published online: 12 October 2015

\section{References}

Angayarkanni SP, Kamal NB, Thavavel V (2012) Automatic detection and classification of cancerous masses in mammogram. In: Third international conference on computing communication and networking technologies

Annamalai M, Guo D, Susan M, Steiner J (2009) An oracle white paper: oracle database $11 \mathrm{~g}$ DICOM medical image support

Artan Y, Haider MA, Langer DL, van der Kwast TH, Evans AJ, Yang Y et al (2012) A boosted Bayesian multiresolution classifier for prostate cancer detection from digitized needle biopsies. IEEE Trans Biomed Eng 59:1205-1218

Benfield MC, Grosjean P, Culverhouse PF, Irigoien X, Sieracki ME, Lopez-Urrutia A et al (2007) RAPID: research on automated plankton identification. Oceanography 20:172-187

Bojar K, Nieniewski M (2008) Mathematical morphology (MM) features for classification of cancerous masses in mammograms, information technologies in biomedicine. Adv Soft Comput 47:129-138. Springer

Camilus KS, Govindan VK, Sathidevi PS (2010) Computer-aided identification of the pectoral muscle in digitized mammograms. J Digit Imaging 23(5):562-580

Elnakib A, Gimel G, Suri JJ, El-baz A, Gimel'farb G (2011) Medical image segmentation: a brief survey. In: El-Baz AS, Acharya UR, Laine AF, Suri JS (eds) Medical image segmentation. Springer, New York, pp 1-39

Erickson BJ, Bartholmai B (2002) Computer-aided detection and diagnosis at the start of the third millennium. J Digit Imaging 15:59-68

Ertas G, Gulcur HO, Aribal E, Semiz A (2001) Feature extraction from mammographic mass shapes and development of a mammogram database, engineering in medicine and biology society. In: Proceedings of the 23rd annual international conference of the IEEE, vol 3, pp 2752-2755

Hassanien AE, Ali JMH (2011) Enhanced rough sets rule reduction algorithm for classification digital mammography. J Intell Syst 13:151-171

Hassanien AE, Badr A (2003) A comparative study on digital a mammography enhancement algorithms based on fuzzy theory. Sci Inform Control 12:21-31 
Jayadevappa D, Srinivas Kumar S, Murty DS (2009) A hybrid segmentation model based on watershed and gradient vector flow for the detection of brain tumor. Int J Signal Process Image Process Pattern Recognit 2(3):29-42

Masek M, Chandrasekhar R, Desilva CJS, Attikiouzel Y (2001), Spatially based application of the minimum cross-entropy thresholding algorithm to segment the pectoral muscle in mammograms. In: The seventh Australian and New Zealand intelligent information systems conference, Nov. 18-21, pp 101-106

Mu T, Nandi AK, Rangayyan RM (2008) Classification of breast masses using selected shape, edge-sharpness, and texture features with linear and kernel-based classifiers. J Digit Imaging 21:153-169

Peng B, Wang Y, Yang X (2010) A multiscale morphological approach to local contrast enhancement for ultrasound images. In: Proceedings of the 2010 international conference on computational and information sciences, ICCIS'10, pp 1142-1145. IEEE Computer Society, Washington, DC

Ramaswamy S, Rose K (2009) Towards optimal indexing for relevance feedback in large image databases. IEEE Trans Image Process 18(12):2780-2789. doi:10.1109/TIP.2009.2028929

Shah H, Ghazali R, Nawi NM (2011) Using artificial bee colony algorithm for MLP training on earthquake time series data prediction. J Comput 3(6):135-142

Thamaraichelvi B, Yamuna GY (2013) A novel efficient kernelized fuzzy C-means with additive bias field for brain image segmentation. In: Proceedings of the international conference on communication and signal processing, pp 68-72

Zakeri FS, Behnam H, Ahmadinejad N (2012) Classification of benign and malignant breast masses based on shape and texture features in sonography. J Med Images Syst 36:1621-1627

Zhang Z, Tan T, Huang K, Wang Y (2012) Three-dimensional deformable-model-based localization and recognition of road vehicles. IEEE Trans Image Process 21(1):1-13

\section{Submit your manuscript to a SpringerOpen ${ }^{\circ}$} journal and benefit from:

- Convenient online submission

- Rigorous peer review

- Immediate publication on acceptance

- Open access: articles freely available online

- High visibility within the field

- Retaining the copyright to your article

Submit your next manuscript at $\boldsymbol{\nabla}$ springeropen.com 